\title{
Effect of Ethanol Addition on the Determination of Thiosulfate Based on Reduction of Ce(IV) and Fluorescence Detection of Ce(III)
}

\author{
Ikko Mikami, ${ }^{\dagger}$ Eri Shibayama, Manami Yuzawa, and Yasuyuki Miura \\ Department of Chemistry, School of Science, Tokai University, 4-1-1 Kitakaname, Hiratsuka 259-1292, Japan
}

\begin{abstract}
The effect of ethanol addition on the determination of thiosulfate based on the reduction of Ce(IV) and fluorescence detection of $\mathrm{Ce}(\mathrm{III})$ was investigated by flow-injection analysis. It was found that the sensitivity of thiosulfate detection was significantly increased by injecting thiosulfate into a mixed solution of $\mathrm{Ce}(\mathrm{IV})$ and ethanol, rather than a solution of $\mathrm{Ce}(\mathrm{IV})$ alone. This is probably due to trace amounts of thiosulfate accelerating the rate of reduction of Ce(IV) by ethanol: $\mathrm{Ce}(\mathrm{IV})$ is slowly reduced by ethanol in the absence of thiosulfate, and thiosulfate serves as a catalyst to the reduction. The detection limits as $S / N=3$ for thiosulfate were very low $\left(10^{-9} \mathrm{M}\right.$ level $)$.
\end{abstract}

(Received June 14, 2012; Accepted August 7, 2012; Published October 10, 2012)

\section{Introduction}

Thiosulfate is a reducing sulfur species found in industrial wastewater, environmental water, human urine, and so on. ${ }^{1-10}$ The determination of thiosulfate is utilized for investigating the chemistry of industrial wastewater, such as from mining sites, ${ }^{1-3}$ offshore oil drilling operations, ${ }^{4}$ or the Kraft process, ${ }^{5}$ and is also of geochemical interest; for example, it can be used to investigate sulfur geochemistry in hydrothermal water. ${ }^{6-8}$ In addition, it is thought to represent a bioindicator of exposure to hydrogen sulfide gas, sulfite oxidase deficiency, or Down's syndrome. ${ }^{9,10}$ Numerous reports on thiosulfate analysis have been published. ${ }^{11-15}$ In these studies, thiosulfate was separated from other species by ion chromatography (IC) or capillary electrophoresis (CE), and detected mainly by measuring the conductivity or absorbance. Several sensitive and selective detection methods have utilized a postcolumn reaction of thiosulfate with $\mathrm{Ce}(\mathrm{IV})$, followed by fluorescence detection of the resulting $\mathrm{Ce}(\mathrm{III}) .{ }^{16-19} \mathrm{Ce}(\mathrm{IV})$ is susceptible to reduction by reducing agents to form $\mathrm{Ce}(\mathrm{III})$, and measurement of the resulting $\mathrm{Ce}$ (III) can allow the determination of the reducing agent. $\mathrm{Ce}$ (III) has a characteristic fluorescence with an excitation maximum at $260 \mathrm{~nm}$ and an emission maximum at $350 \mathrm{~nm}$, while Ce(IV) does not fluoresce. Therefore, Ce(IV) may be employed as a postcolumn reaction agent for the sensitive and selective determination of a reducing agent. This postcolumn reaction has been applied to the IC detection of thiosulfate, polythionates, nitrite, thiosulfate and iodide. ${ }^{16,17}$ Miura et al. ${ }^{18}$ reported that $\mathrm{Ce}(\mathrm{IV})$ can be converted into $\mathrm{Ce}(\mathrm{III})$ by L-ascorbic acid, sulfite, oxalate and thiosulfate in an acidic medium. In these reports, the detection limits for thiosulfate were in the range of $10^{-7}-10^{-6} \mathrm{M}$.

While studying the determination of thiosulfate by fluorescence

† To whom correspondence should be addressed.

E-mail: ikmikami@keyaki.cc.u-tokai.ac.jp detection of $\mathrm{Ce}$ (III), we found that the sensitivity of thiosulfate detection was increased by the addition of a thiosulfate solution into a mixture of $\mathrm{Ce}(\mathrm{IV})$ and alcohol solution, particularly when ethanol was used, compared with a Ce(IV) solution containing no alcohol. We indicated the phenomenon with a batch method. ${ }^{20}$ The purpose of this study was to reveal the effect of alcohol on the determination of thiosulfate with flow injection analysis (FIA) on the assumption of applying to a postcolumn reaction and the cause of this increase in detection sensitivity.

\section{Experimental}

\section{Reagents and chemicals}

All chemicals used were of analytical reagent grade, and were used without further purification. Water was distilled twice, and then purged of dissolved air using an aspirator. A solution of $2.0 \times 10^{-2} \mathrm{M}$ cerium(IV) sulfate in $0.4 \mathrm{M}$ sulfuric acid was prepared by dissolving $1.63 \mathrm{~g}$ of cerium(IV) sulfate tetrahydrate (Kanto Chemical Co., Tokyo, Japan, 99.5\%) in water containing $40 \mathrm{~mL}$ of $2 \mathrm{M}$ sulfuric acid, and diluting the solution to $200 \mathrm{~mL}$ with water. A $4.0 \times 10^{-5} \mathrm{M}$ solution of cerium(IV) sulfate in $0.04 \mathrm{M}$ sulfuric acid, which was used as a reaction solution, was prepared by adding $2 \mathrm{~mL}$ of the $2.0 \times 10^{-3} \mathrm{M}$ cerium(IV) sulfate- $0.4 \mathrm{M}$ sulfuric acid to a $100-\mathrm{mL}$ volumetric flask containing $2 \mathrm{~mL}$ of $2 \mathrm{M}$ sulfuric acid, and diluting it to the mark. A stock solution of thiosulfate (about $0.1 \mathrm{M}$ ) was prepared by dissolving $13.2 \mathrm{~g}$ of sodium thiosulfate pentahydrate (Wako Pure Chemical Industries, Osaka, Japan, 99.0\%) in $500 \mathrm{~mL}$ of water containing a small amount $(0.01 \%)$ of sodium carbonate (Wako Pure Chemical Industries, 99.8\%) as a stabilizer. This solution was standardized by iodometric titration. Working solutions of thiosulfate were obtained by appropriate dilution of the standard solution. Ethanol (99.5\%), methanol (99.8\%), 1-propanol (99.5\%) and 2-propanol (99.7\%) were purchased from Wako Pure Chemical Industries. 


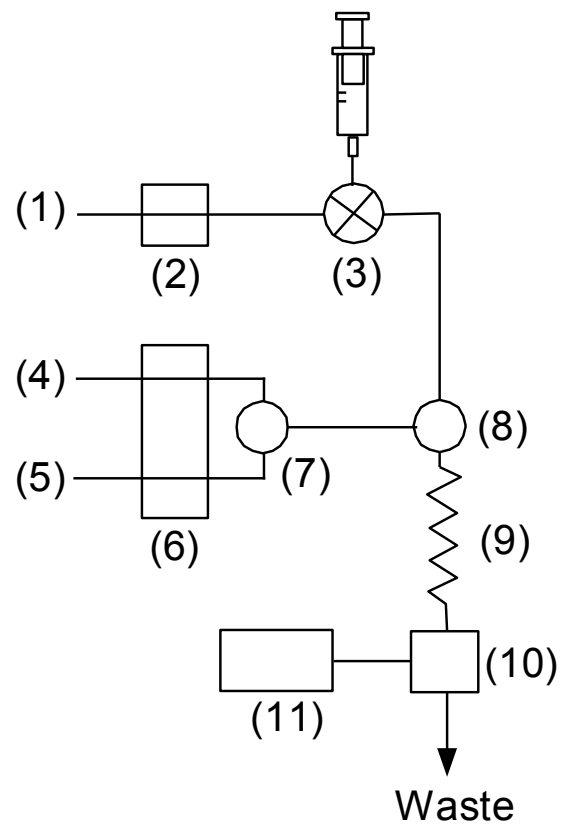

Fig. 1 Flow diagram of the FIA system. (1) Water, (2) pump, (3) injection valve, (4) cerium(IV) sulfate solution, (5) alcohol solution, (6) pump, (7) T-union, (8) mixing tee, (9) reaction tube, (10) fluorescence detector, (11) recorder.

\section{Flow injection method}

A diagram of the FIA system used in this study is shown in Fig. 1. Water, which was used as a carrier, was pumped at a flow rate of $0.40 \mathrm{~mL} \mathrm{~min}^{-1}$ using a LC-10A pump (Shimadzu, Kyoto, Japan). A sample solution containing thiosulfate was injected into the carrier flow by a sample injection valve (Rheodyne, Berkeley, CA) equipped with a $50-\mu \mathrm{L}$ loop. Solutions of $4.0 \times 10^{-5} \mathrm{M}$ cerium(IV) sulfate- $0.04 \mathrm{M}$ sulfuric acid and aqueous alcohol were pumped at a flow rate of $0.20 \mathrm{~mL} \mathrm{~min}^{-1}$ using a DMX-2000 pump (SNK, Tokyo, Japan) and mixed at a T-union to obtain a reaction solution. The reaction solution was then mixed with the carrier solution at a mixing tee.

$\mathrm{Ce}(\mathrm{III})$ formed in the reaction tube $(2 \mathrm{~m} \times 0.5 \mathrm{~mm}$ i.d. $)$ was monitored using a fluorometer (Model RF-10AXL, Shimadzu) at excitation and emission wavelengths of 256 and $354 \mathrm{~nm}$, respectively. The fluorescence intensity of $\mathrm{Ce}$ (III) was recorded using an R-111 recorder (Shimadzu).

\section{Batch method}

Alcohol $(2.5 \mathrm{~mL})$, water $(15.5 \mathrm{~mL})$ and the sample solution $(5.0 \mathrm{~mL})$ containing thiosulfate were placed in a $25-\mathrm{mL}$ volumetric flask. To the flask, $1.0 \mathrm{~mL}$ of $2.5 \times 10^{-3} \mathrm{M}$ cerium(IV) sulfate- $0.25 \mathrm{M}$ sulfuric acid solution was added, and the mixture was immediately diluted to the mark with water. A portion of this reaction mixture was placed in a quartz cell with an optical path length of $1.0 \mathrm{~cm}$; the fluorescence intensity of the resulting $\mathrm{Ce}(\mathrm{III})$ was measured at various standing times after preparation, using a FP-550A spectrofluorometer (JASCO, Tokyo, Japan) at excitation and emission wavelengths of 256 and $354 \mathrm{~nm}$, respectively.

\section{Quantitative analysis of acetaldehyde}

The concentration of acetaldehyde was measured by 2,4-dinitrophenylhydrazine (DNPH) derivatization and HPLC. ${ }^{21}$

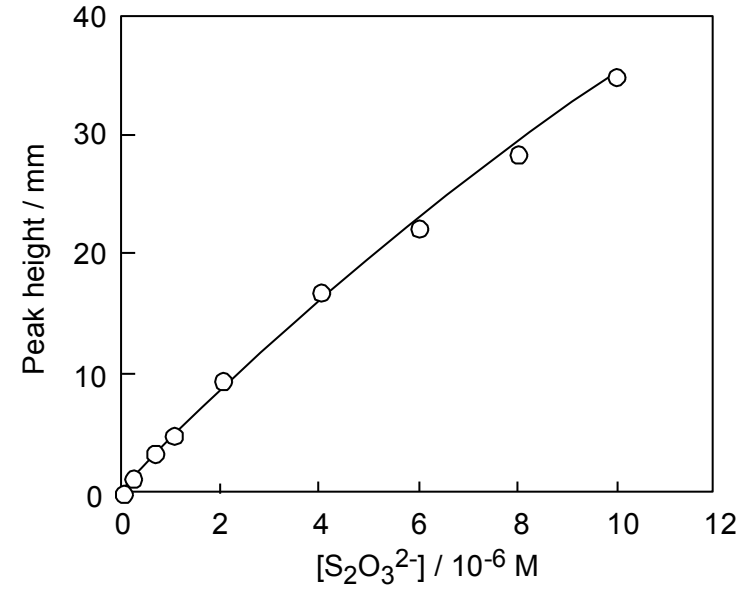

Fig. 2 Calibration plots for thiosulfate obtained by the reaction of thiosulfate with Ce(IV) in the absence of alcohol.

The conditions were as follows: first, a column (Discovery RP Amide C16, $4.6 \mathrm{~mm}$ i.d., $25 \mathrm{~cm}$ length; Sigma-Aldrich) was stabilized by the flow of a $50 \%(\mathrm{v} / \mathrm{v})$ acetonitrile-water solution at $1.0 \mathrm{~mL} \mathrm{~min}^{-1}$ using a L-2130 pump (Hitachi HighTechnologies, Tokyo, Japan) at $313 \mathrm{~K}$, and then a $20-\mu \mathrm{L}$ aliquot of acetaldehyde sample solution containing DNPH, phosphoric acid and glycerine was injected. The absorbance of the resulting acetaldehyde derivative was monitored at $360 \mathrm{~nm}$ using an L-2400 UV detector (Hitachi High-Technologies).

\section{Results and Discussion}

Effect of alcohol addition on the thiosulfate peak height

Figure 2 shows calibration plots for thiosulfate obtained by the FIA method described in the Experimental section, using water instead of an alcohol-water solution. In the absence of alcohol, thiosulfate could be determined at levels as low as $10^{-6} \mathrm{M}$. This is because thiosulfate reduces $\mathrm{Ce}(\mathrm{IV})$ to $\mathrm{Ce}(\mathrm{III}),{ }^{14,16}$ as shown in Eq. (1), and the detection limit for $\mathrm{Ce}$ (III) is around $10^{-6} \mathrm{M}$. The detection limit for thiosulfate under these conditions was calculated as $3.0 \times 10^{-7} \mathrm{M}(S / N=3)$.

$$
2 \mathrm{~S}_{2} \mathrm{O}_{3}{ }^{2-}+2 \mathrm{Ce}(\mathrm{IV}) \longrightarrow \mathrm{S}_{4} \mathrm{O}_{6}{ }^{2-}+2 \mathrm{Ce}(\mathrm{III})
$$

Figure 3 shows the peak heights for $1.0 \times 10^{-7} \mathrm{M}$ thiosulfate in the presence of various concentrations of alcohols (methanol, ethanol, 1-propanol and 2-propanol). The addition of each alcohol to the FIA flow resulted in an increased thiosulfate peak height. Among the alcohols used, ethanol gave the greatest enhancement of thiosulfate peak height; for the rest, the order of enhancement was 2-propanol, 1-propanol and methanol. Although the peak height increased significantly with increases in the ethanol concentration up to $40 \%$, the increase was small over $40-60 \%$. The background noise increased at alcohol concentrations of more than $60 \%$, which made it difficult to detect small thiosulfate peaks.

Calibration plots for thiosulfate upon addition of various alcohols at $40 \%$ are shown in Fig. 4. A linear relationship between the concentration of thiosulfate and its peak height was obtained within the measuring range indicated in the figure. The slope of the calibration plot was greatest for ethanol. In the presence of ethanol at $40 \%$, the detection limit for thiosulfate 


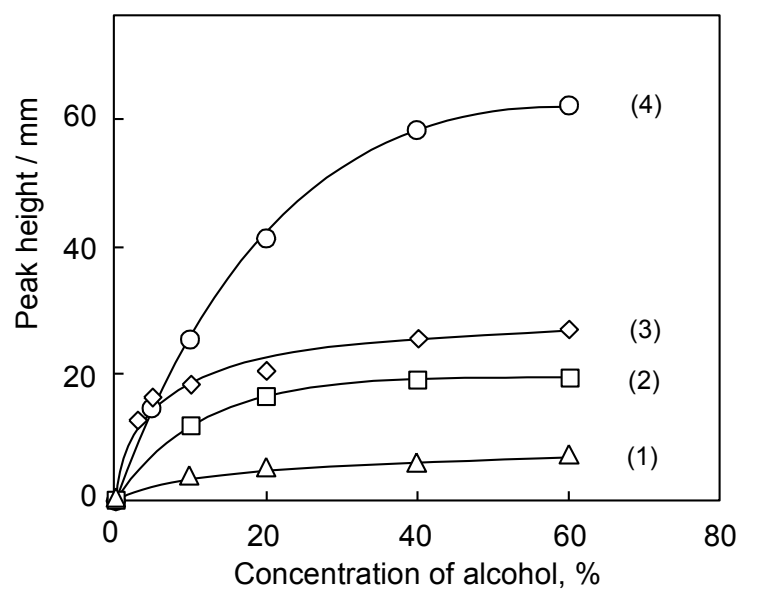

Fig. 3 Effects of alcohols and their concentrations on the peak heights for $1.0 \times 10^{-7} \mathrm{M}$ thiosulfate. (1) Methanol, (2) 1-propanol, (3) 2-propanol, (4) ethanol.

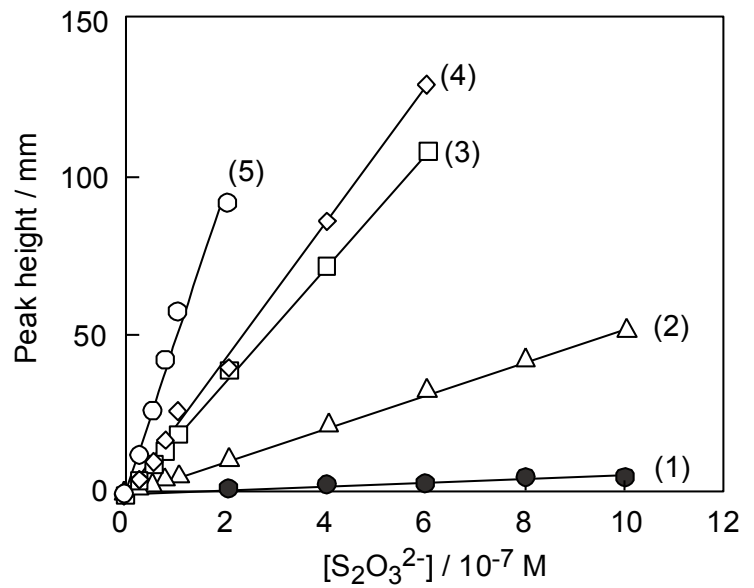

Fig. 4 Calibration plots for thiosulfate in the presence of various alcohols at a concentration of $40 \%$. (1) None, (2) methanol, (3) 1-propanol, (4) 2-propanol, (5) ethanol.

$(S / N=3)$ was found to be $4.6 \times 10^{-9} \mathrm{M}$. This value was quite lower than that obtained with the batch method, ${ }^{20}$ i.e. $1.0 \times 10^{-6} \mathrm{M}$.

Effect of the concentration of $C e(I V)$ on the thiosulfate peak height

Figure 5 shows the influence of the concentration of $\mathrm{Ce}$ (IV) on the peak height for thiosulfate. As can be seen in Fig. 5(a), there was no difference in the slopes of the calibration plots at concentrations of $\mathrm{Ce}(\mathrm{IV})$ below $1.6 \times 10^{-4} \mathrm{M}$. However, the slope gradually decreased as the concentration of $\mathrm{Ce}(\mathrm{IV})$ increased from $1.6 \times 10^{-4}$ to $4.0 \times 10^{-4} \mathrm{M}$. This decrease may be attributed to a decrease in the fluorescence intensity due to partial absorption by the excess $\mathrm{Ce}(\mathrm{IV})$ of emission rays from $\mathrm{Ce}(\mathrm{III})$. At higher thiosulfate concentrations $\left(10^{-6} \mathrm{M}\right)$, shown in Fig. 5(b), plots of the thiosulfate peak height appeared to level off in each $\mathrm{Ce}(\mathrm{IV})$ concentration region, and their peak heights increased in proportion to the concentration of $\mathrm{Ce}(\mathrm{IV})$. The concentration of $\mathrm{Ce}(\mathrm{III})$ at the point of leveling off was estimated from the peak height, and was in agreement with the
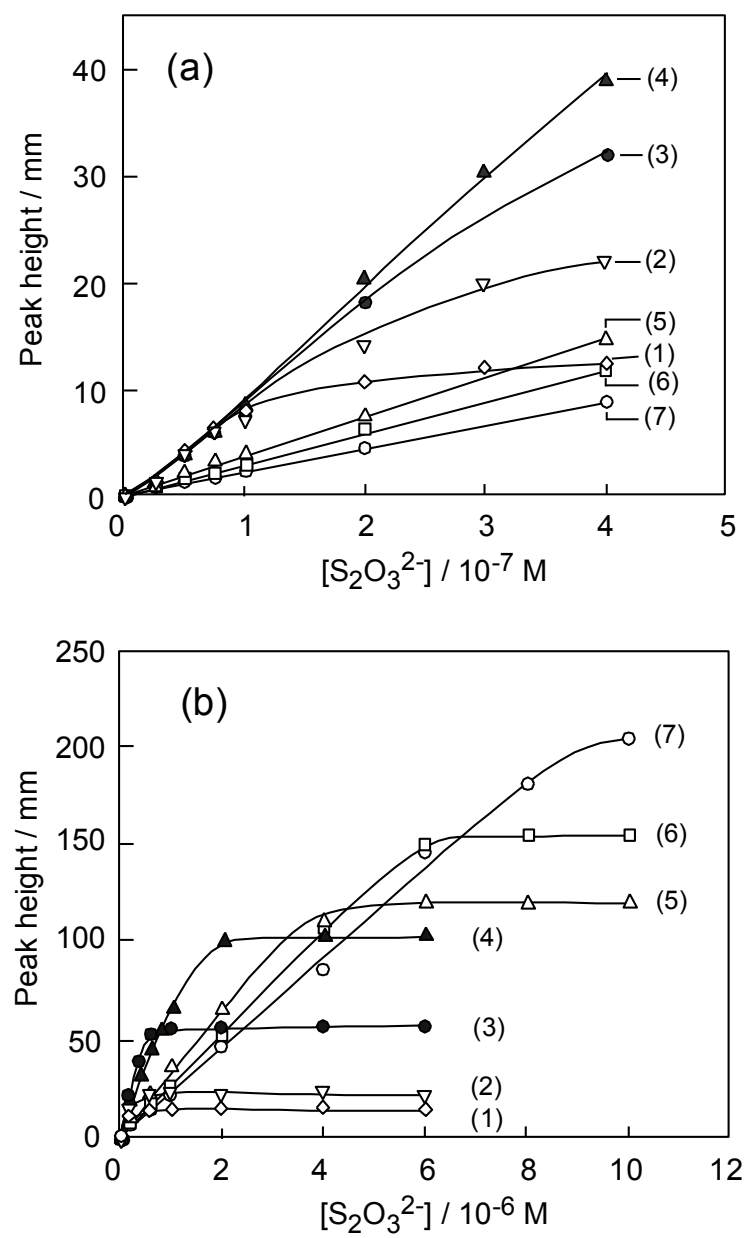

Fig. 5 Effect of the concentration of cerium(IV) sulfate on the peak height of thiosulfate. Concentration of thiosulfate: (a) $0-4.0 \times 10^{-7}$, (b) $0-1.0 \times 10^{-5} \mathrm{M}$. Concentration of cerium(IV) sulfate: (1) $2.0 \times$ $10^{-5}$, (2) $4.0 \times 10^{-5}$, (3) $8.0 \times 10^{-5}$, (4) $1.6 \times 10^{-4}$, (5) $2.4 \times 10^{-4}$, (6) $3.2 \times 10^{-4}$, (7) $4.0 \times 10^{-4} \mathrm{M}$.

concentration of $\mathrm{Ce}(\mathrm{IV})$ in the used reaction solution; that is, $\mathrm{Ce}(\mathrm{IV})$ in the reaction solution was found to be completely converted to $\mathrm{Ce}(\mathrm{III})$. Consequently, sensitive detection of thiosulfate was achieved using $\mathrm{Ce}(\mathrm{IV})$ concentrations below $1.6 \times 10^{-5} \mathrm{M}$. Thiosulfate measurement in a wider concentration range could be carried out using higher concentrations of $\mathrm{Ce}(\mathrm{IV})$, e.g. $4.0 \times 10^{-4} \mathrm{M}$.

Cause of a significant increase in the thiosulfate detection sensitivity upon the addition of ethanol

In order to reveal the cause of the increase in the thiosulfate peak height upon the addition of ethanol, as shown in Figs. 3 and 4 , the fluorescence intensity of a mixture containing thiosulfate and cerium(IV) sulfate was measured over time, using the batch method, in the absence and presence of ethanol.

Figure 6 shows effect of the standing time on the reaction of thiosulfate with $\mathrm{Ce}(\mathrm{IV})$ in the absence of alcohol; the conditions are the same as for the batch method described in the Experimental section, except for the absence of alcohol. The presence of thiosulfate in relatively high concentrations (plots (5) and (6) in Fig. 6) resulted in a slight increase in the fluorescence intensity during in the initial reaction time, but the intensity then remained nearly constant, which implies that the reaction of thiosulfate with $\mathrm{Ce}(\mathrm{IV})$ proceeded rapidly to 


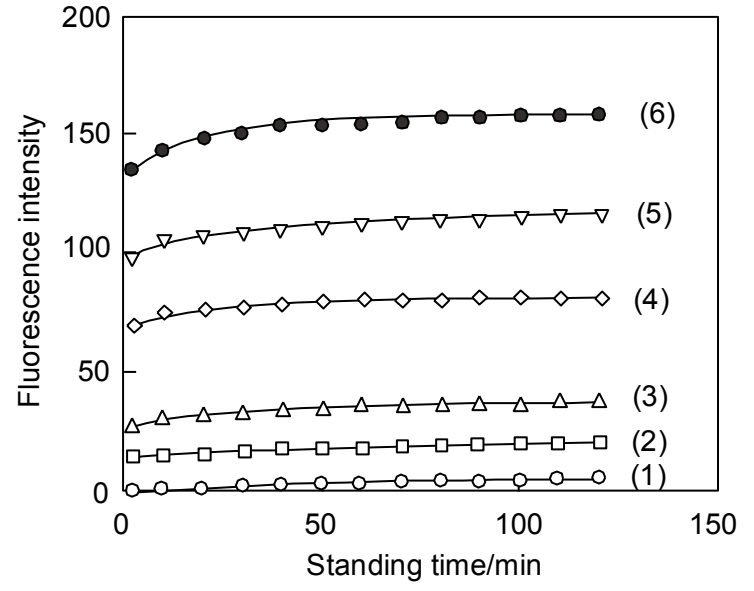

Fig. 6 Time courses of the fluorescence intensity detected using the batch method in the absence of alcohol. Concentration of thiosulfate: (1) 0 , (2) $2.0 \times 10^{-6}$, (3) $4.0 \times 10^{-6}$, (4) $1.0 \times 10^{-5}$, (5) $1.5 \times 10^{-5}$, (6) $2.0 \times 10^{-5} \mathrm{M}$. The concentration of cerium(IV) sulfate was adjusted to $1.0 \times 10^{-4} \mathrm{M}$.

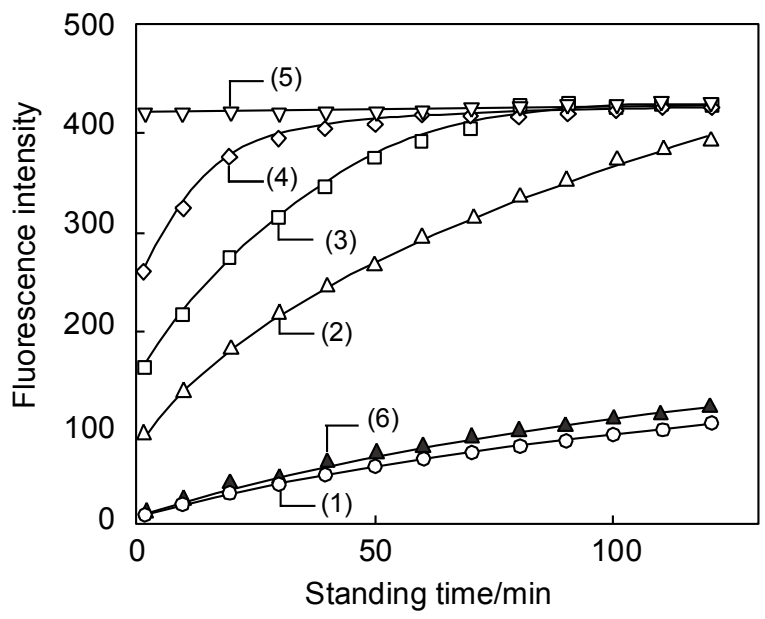

Fig. 7 Time courses of the fluorescence intensity detected using the batch method in the presence of ethanol. Concentration of thiosulfate: (1) 0 , (2) $1.0 \times 10^{-7}$, (3) $2.0 \times 10^{-7}$, (4) $4.0 \times 10^{-7}$, (5) $2.0 \times 10^{-6}$, (6) $1.0 \times 10^{-7} \mathrm{M}$. The concentrations of ethanol and cerium(IV) sulfate were adjusted to $1.8 \mathrm{M}(10 \mathrm{vol} \%)$ and $1.0 \times 10^{-4} \mathrm{M}$, respectively. The mixing order for each reaction is described in the Experimental section, except for (6); in that case, thiosulfate solution and cerium(IV) sulfate solution were mixed, and then ethanol was added.

completion. The fluorescence intensity obtained after long reaction times (for example, $60 \mathrm{~min}$ ) varied in proportion to the thiosulfate concentration, with thiosulfate thought to be oxidized completely by $\mathrm{Ce}(\mathrm{IV})$.

Plot (1) in Fig. 7 shows the fluorescence intensity of a mixture of cerium(IV) sulfate and ethanol plotted against the standing time, with the concentrations of cerium(IV) sulfate and ethanol adjusted to $1.0 \times 10^{-4} \mathrm{M}$ and $1.8 \mathrm{M}(10 \%)$, respectively. The fluorescence intensity gradually increased with the standing time, which shows that the $\mathrm{Ce}(\mathrm{IV})$ is slowly reduced by the ethanol. In the case of a mixture of cerium(IV) sulfate and thiosulfate (the concentrations of cerium(IV) sulfate and thiosulfate were adjusted to $1.0 \times 10^{-4}$ and $1.0 \times 10^{-7} \mathrm{M}$, respectively), no fluorescence was detected, since the concentration of thiosulfate was quite low. However, plot (2) shows that for a mixture of cerium(IV) sulfate, ethanol and thiosulfate (with concentrations adjusted to $1.0 \times 10^{-4} \mathrm{M}, 1.8 \mathrm{M}$ $(10 \%)$ and $1.0 \times 10^{-7} \mathrm{M}$, respectively), the fluorescence intensity increased significantly in comparison with plot (1). Taking into account the relatively low thiosulfate concentration, it seems that thiosulfate catalytically accelerates the rate of the reaction between Ce(IV) and ethanol. The fluorescence intensity at short standing times (for example, $2 \mathrm{~min}$ ) increased with increasing the thiosulfate concentration, as can be seen in plots (2) - (5). Therefore, it was concluded that highly sensitive detection of thiosulfate, as shown in Fig. 4, could be achieved as a result of an increase in the reduction rate of $\mathrm{Ce}$ (IV) by ethanol in the presence of thiosulfate, which plays a catalytic role. An increase in the fluorescence intensity at about $420 \mathrm{~nm}$ was not observed in plots (3) - (5) due to complete consumption of the Ce(IV) used in the reaction solution.

Plot (6) in Fig. 7 was obtained using the same concentrations of $\mathrm{Ce}(\mathrm{IV})$, ethanol and thiosulfate as for plot (2), but the mixing order was varied from that described in the Experimental section; in this case, the thiosulfate solution and cerium(IV) sulfate solution were mixed first, and then ethanol was added. Despite the fact that the concentration of each component was the same as for plot (2), the rate of increase of fluorescence intensity in plot (6) was considerably lower than that in plot (2), and was similar to that shown in plot (1), which was obtained in the absence of thiosulfate. This is due to the fact that oxidation of thiosulfate by $\mathrm{Ce}(\mathrm{IV})$ proceeds quickly in the absence of ethanol. These results also indicate that ethanol prevents the oxidation of thiosulfate by $\mathrm{Ce}(\mathrm{IV})$, which means that thiosulfate can remain in the solution for a longer period, playing a catalytic role.

Figure 3 indicates that the presence of ethanol resulted in a more sensitive determination of thiosulfate compared with methanol, 1-propanol and 2-propanol. This is thought to be because the rate of reduction of cerium by ethanol is the lowest among the four alcohols,$^{20}$ and the increase in the reduction rate due to addition of thiosulfate is the greatest in the case of ethanol.

Products formed by oxidation of ethanol with $\mathrm{Ce}(I V)$ in the presence of thiosulfate

Next, we examined the products formed by the oxidation of ethanol with $\mathrm{Ce}(\mathrm{IV})$, accelerated catalytically by thiosulfate. We measured the concentration of acetaldehyde in a mixture of cerium(IV) sulfate $\left(1.0 \times 10^{-4} \mathrm{M}\right)$, ethanol $(1.8 \mathrm{M})$ and thiosulfate $\left(1.0 \times 10^{-7} \mathrm{M}\right)$ by the DNPH-HPLC method. When the mixture was allowed to stand for $1 \mathrm{~h}$, acetaldehyde was detected at $1.1 \times 10^{-5} \mathrm{M}$. At this time, the concentration of $\mathrm{Ce}(\mathrm{III})$ formed was $2.2 \times 10^{-5} \mathrm{M}$. Therefore, acetaldehyde and $\mathrm{Ce}$ (III) were formed at a molar ratio of 1:2. This ratio was in accordance with the stoichiometric proportions of Eq. (2).

$$
2 \mathrm{Ce}(\mathrm{IV})+\mathrm{C}_{2} \mathrm{H}_{5} \mathrm{OH} \longrightarrow 2 \mathrm{Ce}(\mathrm{III})+\mathrm{CH}_{3} \mathrm{CHO}+2 \mathrm{H}^{+}
$$

Although capillary electrophoresis was used to investigate whether acetic acid was present in the sample, it was not detected. When an acetaldehyde solution $\left(2.0 \times 10^{-5} \mathrm{M}\right)$ was introduced into the FIA flow instead of an alcohol solution, the fluorescence intensity for thiosulfate was the same as that obtained in the absence of acetaldehyde. Therefore, thiosulfate accelerates the reaction shown in Eq. (2), and the resulting acetaldehyde does not affect the fluorescence intensity. 


\section{Conclusions}

In the determination of thiosulfate by the fluorescence detection of $\mathrm{Ce}$ (III), the fluorescence intensity was highly enhanced by the addition of alcohols, such as ethanol, methanol, 1-propanol and 2-propanol into the FIA flow. The addition of ethanol resulted in the greatest enhancement of the fluorescence intensity. While ethanol slowly reduced $\mathrm{Ce}(\mathrm{IV})$ to form acetaldehyde and $\mathrm{Ce}$ (III) in the absence of thiosulfate, the reduction rate increased dramatically in the presence of thiosulfate, even when the concentration of thiosulfate was quite low. Thus, thiosulfate enhanced the reduction rate catalytically, and the reduction rate increased in line with the concentration of thiosulfate.

The type of alcohol added and its concentration, as well as the concentration of $\mathrm{Ce}(\mathrm{IV})$, were found to affect the detection limit and measurable concentration range of thiosulfate. The detection limit $(S / N=3)$ for thiosulfate was $4.6 \times 10^{-9} \mathrm{M}$ in a reaction solution of $40 \%$ ethanol and $4.0 \times 10^{-5} \mathrm{M} \mathrm{Ce}(\mathrm{IV})$ sulfate, using the FIA technique. This reaction is expected to be applicable to the detection of extremely low concentrations of thiosulfate as a postcolumn reaction. This relatively high sensitivity allows the detection of thiosulfate in sample solutions at higher dilution rates, which is expected to diminish the influence of co-existing species on thiosulfate detection.

\section{References}

1. M. G. Aylmore and D. M. Muir, Miner. Eng., 2001, 14, 134.

2. M. I. Jeffrey and S. D. Brunt, Hydrometallurgy, 2007, 89, 52.

3. A. C. Grosse, G. W. Dicinoski, M. J. Shaw, and P. R.
Haddad, Hydrometallurgy, 2003, 69, 1.

4. H. S. Kalal, M. Ghadiri, A. A. M. Beigi, and S. A. S. Sadjadi, Anal. Chim. Acta, 2004, 502, 133.

5. S. Rabin and J. Stillian, J. Chromatogr., A, 1994, 671, 63.

6. M. B. Goldhaber, Am. J. Sci., 1983, 238, 193.

7. H. Fossing and B. B. Jorgensen, Geochim. Cosmochim. Acta, 1990, 54, 2731.

8. Y. Xu, M. A. A. Schoonen, D. K. Nordstrom, K. M. Cunningham, and J. W. Ball, Geochim. Cosmochim. Acta, 1998, 62, 3729.

9. G. Chwatko and E. Bald, Talanta, 2009, 79, 229.

10. T. Togawa, M. Ogawa, M. Nawata, Y. Ogasawara, K. Kawanabe, and S. Tanabe, Chem. Pharm. Bull., 1992, 40, 3000 .

11. J. W. O'Reilly, G. W. Dicinoski, M. J. Shaw, and P. R. Haddad, Anal. Chim. Acta, 2001, 432, 165.

12. A. Padarauskas, V. Paliulionyte, R. Ragauskas, and A. Dikcius, J. Chromatogr., A, 2000, 879, 235.

13. L. M. de Carvalho and G. Shwedt, J. Chromatogr., A, 2005, 1099, 185.

14. C. O. Moses, D. K. Nordstrom, and A. L. Mills, Talanta, 1984, 31, 331.

15. S. Motellier and M. Descostes, J. Chromatogr., A, 2001, 907, 329.

16. A. W. Wolkoff and R. H. Larose, Anal. Chem., 1975, 47, 1003.

17. S. H. Lee and L. R. Field, Anal. Chem., 1984, 56, 2647.

18. Y. Miura, M. Hatakeyama, T. Hosino, and P. R. Haddad, $J$. Chromatogr., A, 2002, 956, 77.

19. D. P. Kelly and A. P. Wood, Method in Enzymology, 1994, $243,475$.

20. I. Mikami and Y. Miura, Proc. Sch. Sci. Tokai Univ., 2008 43, 179.

21. K. Fung and D. Grosjean, Anal. Chem., 1981, 53, 168. 